\title{
Quality of life patients treated with rituximab intravenous vs. subcutaneous in B-cell lymphomas: results from outpatient Hematooncology Clinic in Lodz
}

\author{
Magdalena Witkowska1, Sonia Witkowska², Marika Klimczak², Anna Frydecka², \\ Michał Witkowski ${ }^{3}$, Piotr Smolewski ${ }^{1}$ \\ 1 Department of Experimental Hematology, Medical University of Lodz, Poland \\ Copernicus Memorial Hospital \\ 2 Medical University of Lodz, Poland \\ ${ }^{3}$ Copernicus Memorial Hospital, Lodz, Poland
}

Correspondence: Magdalena Witkowska Department of Experimental Hematology, Medical University of Lodz Copernicus Memorial Hospital, Poland

93-510 tódź, Ciołkowskiego 2 e-mail:magdamalicka@gmail.com phone: 426895191 fax: 426895192

Received: 11.05.2021 Accepted: 25.06.2021

DOI: 10.24292/01.OR.1222550621 Copyright $\odot$ Medical Education. All rights reserved.

\section{ABSTRACT}

Introduction: Nowadays, rituximab is available both as intravenous (IV) and subcutaneous (SC) formulations. The aim of this study is to compare quality of life (QOL) of patients treated in Hematooncology Clinic in Lodz with B-cell non-Hodgkin lymphoma (B-NHL) treated with rituximab IV and SC. Material and methods: In 50 adult patients with B-NHL diagnosis we assessed QOL by three different questionnaires (EORTC QLQ-C30, FACT/GOG-NTx and EQ-5D). We compared it between patients treated with rituximab IV and SC.

Results: In comparison of general condition in EQ-5D-3L and EORTC QLQ-C30 questionnaire patients treated with rituximab SC felt statistically better than with IV administration $(p<0.01)$. In SC group general health was evaluated for 72 while in IV for only 58.1. According to FACT/GOG-NTx questionnaire SC group had significantly better physical well-being. In SC group we observed statistically more complete responses (CR) 21 (88\%) vs. 17 (65\%) in IV group. Moreover, trend was observed in emotional well-being in favor of SC treatment.

Discussion: In our study we observed for the first time in the literature statistical difference in frequency of vomiting, nausea, and lack of appetite mentioned from our patients in questionnaire. It is very important that this findings were not published in any other study comparing SC and IV route od administration. Also looking at economic side of SC administration, this type of dosing. As a result it should be preferred form for B-cell lymphoma.

Key words: rituximab, quality of life, anti CD20, B-cell NHL, lymphoma 


\section{INTRODUCTION}

Rituximab is a chimeric monoclonal antibody against CD20 transmembrane antigen which is located on the B-cell surface. As the result of antibody binding, $\mathrm{B}$-cells die by mechanisms such as antibody-dependent cell-mediated cytotoxicity, complement-dependent cytotoxicity and apoptosis [1]. That is why rituximab as monotherapy or combined with cyclophosphamide, doxorubicin, vincristine, and prednisolone (R-CHOP) became the standard of care for B-cell non-Hodgkin lymphomas (NHLs). Clinical studies have shown that rituximab given as a single agent or in combination with chemotherapy prolongs progression-free survival (PFS) and overall survival (OS) [2]. It also has a well-established safety profile and a wide therapeutic window [3]. Successful clinical use of rituximab has led to the development of other anti-CD20 monoclonal antibodies, like obinutuzumab, ofatumumab or veltuzumab [4].

For many years only intravenous (IV) administration of rituximab has been approved for therapeutic purposes. Nowadays, it is available both as IV and subcutaneous (SC) formulations. The SC formulation combines the original formula of rituximab with recombinant human hyaluronidase $(\mathrm{rHuPH} 20)$ which increases the permeability of tissue through transient depolymerization of hyaluronic acid. It enables to administrate SC in larger volumes. The enzyme enhances bioavailability of locally injected large protein therapeutics [5]. These forms of rituximab differ in concentration and dosing. This difference has an influence on time of administration, costs of medical procedures and also the reaction of a patient's organism. Studies has demonstrated that SC rituximab has similar durability, pharmacodynamics, and efficacy compared with IV rituximab [6]. By the SABRINA trial (NCT01200758) both forms have proven to be equally safe and have the same level of clinical activity [7].

The SC formulation occurs as specified dose in a ready to use vial with estimated administration time at 5 minutes. The SC may be used to mitigate the risk of hematological toxicities and/or other chemical mediators and reduce the burden of treatment by shortening the time spent at a care unit and facilitating the procedure of preparation and administration of the medicine. Compared with rituximab IV, SC formulation was associated with reduced chair time and active healthcare professional time [8] All of these factors could lower the costs and ease patients to endure the treatment [9].

The aim of this study is to compare quality of life (QOL) of patients treated in Hematooncology Clinic in Lodz with B-cell lym- phoma treated with rituximab IV and SC and verify that there are fewer side effects when SC formulation is applied.

\section{MATERIALS AND METHODS}

\section{Study design}

This study was conducted from June 2019 to February 2020 at day care unit of Hematology Clinic in Lodz, Poland. We assessed the QOL in B-cell lymphoma patients treated with rituximab IV vs. SC. At out-patient clinics we explained the study to participants and obtained informed consent. The patients were asked to answer the survey questionnaire before they left the clinic.

\section{Patients}

There were 50 patients enrolled to this study. Inclusion criteria were patients who were diagnosed with B-cell non-Hodgkin lymphoma (NHL) including diffuse large B-cell lymphoma (DLBCL), mantle cell lymphoma $(\mathrm{MCL})$ and grade $3 \mathrm{~B}$ follicular lymphoma (FL) according to the World Health Organization criteria. Patients were treatment naïve disease, were capable of answering questionnaires at the time of the survey and age 18 years or older. All patients had grade 0 or 1 according to Eastern Cooperative Oncology Group (ECOG). All pathology was reviewed by a lymphoma hematopathologist. Patients were treated with therapeutic purpose of cyclophosphamide, doxorubicin, vincristine and prednisone $\mathrm{CHOP}$-based chemotherapy with rituximab IV or SC. Patients were included into IV or SC group according to doctors decision. The study group characteristic is shown in table 1.

\section{Data collection}

Medical records of each patient included in this study were collected form available documents, clinical and histopathological results from the Hospital Electronic Health Record System.

Data about QOL were obtained from three types of questionnaires after $6^{\text {th }}$ course of treatment which are:

1. EORTC QLQ-C30 (version 3.0)

2. FACT/GOG-NTx (version 4)

3. EQ-5D (Polish version for Poland).

The EORTC QLQ-C30 scale is made of five multi-item functional scales that evaluate physical, role, emotional, cognitive, and social function and one global health status/QoL scale. Three symptom scales measure fatigue, pain, and nausea/vomiting, and six single items assess other symptoms (dyspnea, insomnia, appetite loss, constipation, and diarrhea) and financial difficulties. 
TABLE 1.

Study group characteristics.

\begin{tabular}{|c|c|c|}
\hline Number of patients & \multicolumn{2}{|c|}{50} \\
\hline \multirow{2}{*}{ Gender } & women & 28 \\
\hline & men & 22 \\
\hline Median age (years) & \multicolumn{2}{|c|}{$67(31-94)$} \\
\hline \multirow{3}{*}{ Histopatology } & DLBCL & 35 \\
\hline & $\mathrm{MCL}$ & 8 \\
\hline & $\mathrm{FL}$ & 7 \\
\hline \multirow{2}{*}{ Type of administration } & SC & 24 \\
\hline & IV & 26 \\
\hline \multirow{4}{*}{$\begin{array}{c}\text { Lugano } \\
\text { classification }\end{array}$} & 1 & 13 \\
\hline & $\|$ & 10 \\
\hline & III & 12 \\
\hline & IV & 15 \\
\hline \multirow{2}{*}{ B symptoms } & no & 27 \\
\hline & yes & 23 \\
\hline Nodal & \multicolumn{2}{|c|}{32} \\
\hline \multirow{7}{*}{ Extranodal } & & stomach 2 \\
\hline & & large intestine 4 \\
\hline & & liver 2 \\
\hline & & tongue 1 \\
\hline & & sinuses 2 \\
\hline & & nasopharyngeal 2 \\
\hline & & adenoid 5 \\
\hline \multirow{3}{*}{ I-line therapy } & $\mathrm{R}-\mathrm{CHOP}$ & 43 \\
\hline & miniRCHOP & 3 \\
\hline & RCOP & 4 \\
\hline \multirow{4}{*}{ Treatment response } & $\mathrm{CR}$ & 38 \\
\hline & PR & 2 \\
\hline & SD & \\
\hline & PD & 10 \\
\hline \multirow{3}{*}{ II-line therapy } & HD-AraC & 1 \\
\hline & $\mathrm{BR}$ & 4 \\
\hline & ICE & 6 \\
\hline AutoSCT & \multicolumn{2}{|c|}{2} \\
\hline Deaths & \multicolumn{2}{|c|}{5} \\
\hline
\end{tabular}

IV - intravenous; autoSCT - autologous stem-cell transplantation; BR - bendamustine + rituximab; CR - complete response; DLBCL - diffuse large B-cell lymphoma;

$\mathrm{FL}$ - follicular lymphoma; HD-AraC - high dose cytarabine; ICE - ifosfamide + carboplatin + etoposide; MCL - mantle cell lymphoma; miniRCHOP - reduced dose rituximab + cyclophosphamide + doxorubicin + vincristine + prednisone; PD - progressive disease; PR - partial response; R-CHOP - rituximab + cyclophosphamide + doxorubicin + vincristine + prednisone; SC - subcutaneous; SD - stable disease.

We scored the EORTC QLQ-C30 items according to a scoring manual, and the data was linearly transformed to yield scores from 0 to 100 . Higher scores indicate a better status in functioning domains but a worse status in symptom domains. The threshold for impaired function and symptoms that negatively affect QOL were 66 and 33, respectively.

We also evaluated Functional Assessment of Cancer Therapy-General scale (FACT- GOG-NTx, version 4) a general QOL assessment for cancer patients which measures four QOL domains: physical, social/family, emotional, and functional well-being (WB). Patients rate 27 items from $0-4$, " 4 " reflecting items that "very much" apply to them in the past 7 days. The four subscales are added to calculate an overall QOL score, and a lower score indicates a poorer QOL. Possible FACT/GOG-NTx scores are: 0-24 for emotional WB, 0-28 for other WB subscales, and 0-108 for total WB. Previous evaluations of the FACT/GOG-NTx have reported strong reliability and validity, including in lymphoma patients [7].

The EuroQol EQ-5D was developed by an international, multidisciplinary group of researchers in adult patients to establish a standardized generic instrument capable of being expressed as a single index value. It is made up of two components: a description of the patient's health status and a self-rating of their current status, using a visual analogue scale (VAS). The VAS is a vertical thermometer anchored by the "best imaginable health state" 
at the top of the scale (100) and the "worst imaginable health state" at the bottom (0).

All of three questionnaires were fully explained to obtain full understanding of the task by the patient. Each patient answered and completed four questionnaires in one day during stay in the Chemotherapy Department of the Hospital.

\section{Statistical analysis}

Subject characteristics and scores of EORTC QLQ-C30, FACT/ GOG-NTx, EQ-5D-3L and FACIT were summarized using mean \pm standard deviation for continuous variables and number (\%) for categorical variables. Characteristics of each patients group were compared using ANOVA for continuous variables and chi-square tests for categorical variables. We conducted multivariable linear regression to evaluate the association of questionnaires in $\mathrm{NHL}$ patients with age at diagnosis (continuous), sex (male or female), disease type (aggressive and indolence), and length of survival (continuous). $p$-values of $<0.05$ were considered significant, and two-sided tests were used in all calculations. Statistical analyses were performed using STATA ver. 13.0 statistical software (Stata Corp LP, College Station, TX) and IBM PASW ver. 18.0 package (SPSS Inc., Chicago, IL).

\section{Ethical statement}

This study was conducted in accordance with the Declaration of Helsinki and the International Conference on Harmonization guidelines for Good Clinical Practice. All patients gave written informed consent. The protocol was approved by the local ethics committees.

\section{RESULTS}

In total 50 patients with B-cell NHL received either rituximab SC $(n=24)$ or IV $(n=26)$ in the study. In the SC group there were 23 patients with DLBCL diagnosis and 1 with FL. In IV study 12 patients had DLBCL, $6 \mathrm{FL}$ and $8 \mathrm{MCL}$. Baseline demographic and disease characteristics were well-balanced between both (tab. 2).

\section{Demographics, disease and treatment characteristics} The date of initial diagnosis ranged from May 2005 to March 2019. The sample was all white with a median (SD) age of $64 \mathrm{vs.}$ 67 years old in IV and SC accordingly. In SC group there were 7 man (29\%) and 15 (58\%) in IV group. The majority of patients had DLBCL diagnosis in both groups - $23(68 \%)$ in SC group and $16(62 \%)$ in IV group. All disease and treatment characteristics are shown in table 2 . There were no statistical difference between groups in age, gender, race or disease stage. ECOG status was $0-1$ in all patients and did not significantly differ across treatment groups. There were no statistical difference in B symptoms and stage of disease according to Lugano classification. The only difference between both group were observed in extranodal disease (not isolated). In SC group 50\% patients has extranodal lymphoma involvement vs. $23 \%$ in IV group $(p<0.01)$.

TABLE 2.

Comparison of SC and IV group.

\begin{tabular}{|c|c|c|}
\hline Type of administration & SC & IV \\
\hline $\begin{array}{l}\text { Nodal vs. } \\
\text { extranodal }\end{array}$ & $\begin{array}{l}12(50 \%) \\
12(50 \%)\end{array}$ & $\begin{array}{c}20(77 \%) \\
6(23 \%)\end{array}$ \\
\hline $\begin{array}{l}\text { Lugano classification } \\
\text { - I } \\
\text { - II } \\
\text {. III } \\
\text {. IV }\end{array}$ & $\begin{array}{c}10(42 \%) \\
1(4 \%) \\
3(12 \%) \\
10(42 \%)\end{array}$ & $\begin{array}{l}3(12 \%) \\
9(35 \%) \\
9(35 \%) \\
5(18 \%)\end{array}$ \\
\hline B symptoms & $12(50 \%)$ & $16(61 \%)$ \\
\hline $\begin{array}{l}\text { Surgical treatment } \\
\text { Radiotherapy }\end{array}$ & $\begin{array}{l}2(8 \%) \\
1(4 \%)\end{array}$ & $\begin{array}{c}1(4 \%) \\
0\end{array}$ \\
\hline $\begin{array}{l}\text { I-line treatment } \\
\text { - R-CHOP } \\
\text { - miniRCHOP } \\
\text { - RCOP }\end{array}$ & $\begin{array}{c}21(88 \%) \\
1(4 \%) \\
2(8 \%)\end{array}$ & $\begin{array}{c}22(84 \%) \\
2(8 \%) \\
2(8 \%)\end{array}$ \\
\hline $\begin{array}{l}\text { Treatment response } \\
\text { - CR } \\
\text { - PR } \\
\text { - SD } \\
\text { - PD } \\
\end{array}$ & $\begin{array}{c}21(88 \%) \\
2(8 \%) \\
0 \\
1(4 \%)\end{array}$ & $\begin{array}{c}17(65 \%) \\
0 \\
0 \\
9(35 \%) \\
\end{array}$ \\
\hline Relapsed and refractory & $2(8 \%)$ & $9(35 \%)$ \\
\hline Deaths & $1(4 \%)$ & $4(15 \%)$ \\
\hline
\end{tabular}

IV - intravenous; $C R$ - complete response; miniRCHOP - reduced dose rituximab + cyclophosphamide + doxorubicin + vincristine + prednisone; $P D$ - progressive disease $\mathrm{PR}$ - partial response; $\mathrm{SC}$ - subcutaneous; $\mathrm{SD}$ - stable disease; $\mathrm{R}-\mathrm{CHOP}$ - rituximab + cyclophosphamide + doxorubicin + vincristine + prednisone; $\mathrm{RCOP}$ - rituximab + cyclophosphamide + vincristine + prednisone. 
In both treatment group majority of patients obtained rituximab in combination with cyclophosphamide, doxorubicin, vincristine and prednisone (CHOP), 21 (88\%) in SC group and 22 (84\%) in IV group. Reduced dose CHOP (miniCHOP) was given to 3 patients 1 in SC group and 2 in IV. 4 patients were treated with cyclophosphamide, vincristine and prednisone (COP) due to cardiovascular disease, 2 in both groups. The treatment response differed significantly across both treatment groups. Patients who received rituximab SC had much better outcome than patients with IV administration. In SC group we observed statistically more complete responses (CR) 21 (88\%) vs. 17 (65\%) in IV group $(p<0.01)$. Moreover, in IV group there were statistically more patients with progressive disease (PD) 9 (35\%) vs. only $1(4 \%)$ in SC group. Statistically more relapsed and refractory disease - 2 (8\%) vs. 9 (35\%) and deaths - 1 (4\%) vs. 4 (15\%) were observed in IV group $(p<0.01)$. PFS was not reached in both groups. The time of observation was 18 months.

\section{QUALITY OF LIFE ASSESSMENT}

Overall 50 patients completed all three questionnaire on day first of sixth course (EORTC QLQ-C30, FACT/GOG-NTx and EQ-5D-3L) including 24 patients from SC group and 26 from IV group. In comparison of general condition in EQ-5D-3L questionnaire patients treated with rituximab SC felt statistically better than patients who received this agent IV $(p<0.01)$. In SC group general health was evaluated by patients for 72 while in IV group for only 58.1 (fig. 1). According to FACT/GOG-NTx questionnaire patients in SC group had significantly better physical well-being and less additional cancers (fig. 2). Moreover, trend was observed in emotional well-being in favor of SC treatment. There were no difference observed in social and functional well-being. In the last EORTC QLQ-C30 questionnaire in SC group both general health condition and evaluation of $\mathrm{QOL}$ was significantly better $(p<0.01)$ than in IV group (fig. 3). Patients in SC group had less gastric problems including vomiting, lack of appetite and nausea than in IV group. Moreover, there were trend that easy fatigue was observed more often in IV group.

\section{DISCUSSION}

There is an increasing evidence supporting the use of rituximab SC instead of IV in patients with different B-cell NHL. Moreover, extremely important is the potential impact of this therapy on QOL. The current study abstracted data from Hematooncology Clinic in Copernicus Hospital in Lodz, Poland. The population selected in SC and IV groups were similar in age, gender, NHL type and ECOG status.
FIGURE 1.

QOL by EQ-5D-3L in lymphoma patients treated with rituximab administered SC $(n-26)$ vs. IV $(n-24)$.

\section{EQ-5D-3L}

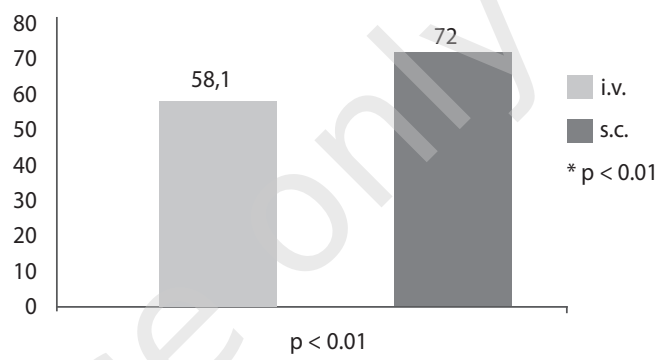

FIGURE 2.

QOL assessed by FACT/GOG-Ntx in lymphoma patients treated with rituximab administered SC ( $n-26)$ vs. IV $(n-24)$.

\section{FACT/606-Ntx}

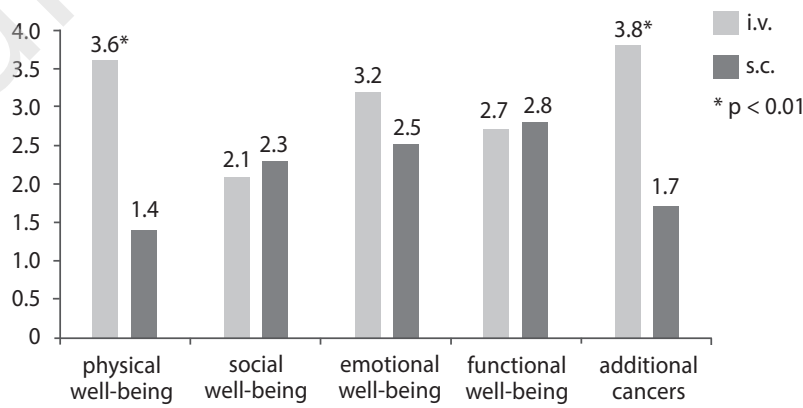

FIGURE 3.

QOL assessed by and EORT-QLQ-C310 in lymphoma patients treated with rituximab administered SC $(n-26)$ vs. IV $(n-24)$.

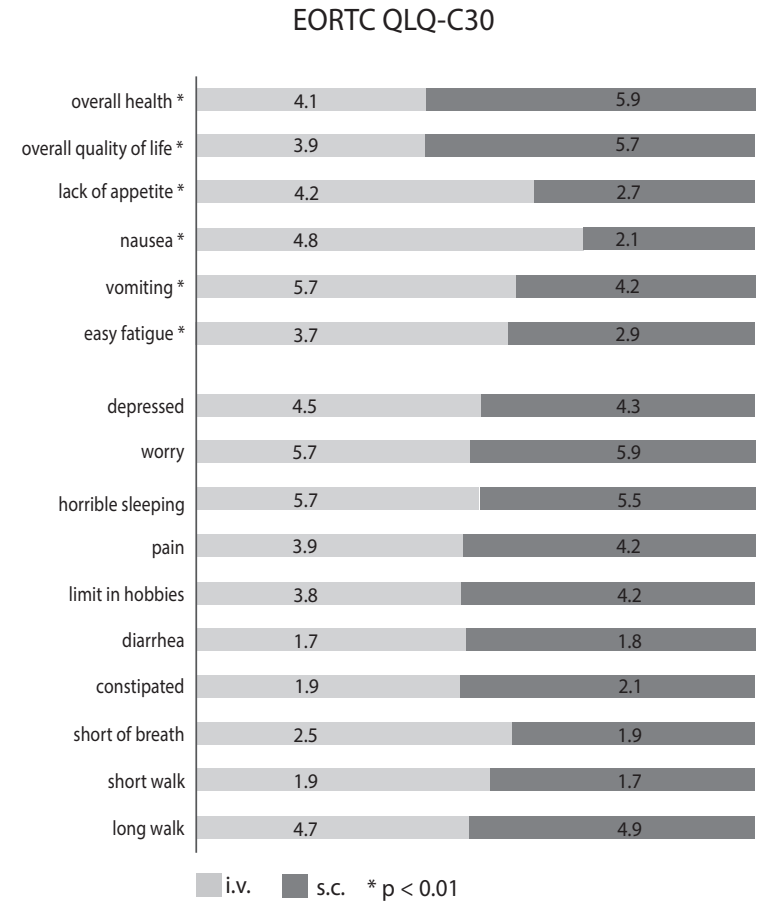


Our findings showed show, strong patients preference for SC administration of rituximab than IV. Rituximab was administered in both arms as a part of IV chemotherapy component. According to our study he majority of patients in a comment unit of questionnaire mentioned that SC dosing "requires less time in hospital" and "is more comfortable during administration". Similar results were observed in studies comparing SC and IV administration of transtuzumab in HER-2 positive breast cancer patients $[10,11]$. Subcutaneous trastuzumab via the single-use injection device was preferred by 216 patients ( 91.5 vs. 95\%; $p<0.0001$ ). Only 16 patients preferred intravenous trastuzumab (6.8\%), and four had no preference (1.7\%). In both studies, our and PrefHer, patients with SC treatment were more satisfied, adverse events occurred less often and were less serious.

Data from our study showed that overall treatment satisfaction and overall health in EQ-5D-3L and FACT/GOG-Ntx questionnaires was higher for SC vs. IV administration. The advantage of our study was using three different types of questionnaire. As a result, we were able to assess the $\mathrm{QOL}$ more accurately than using just one of them. Our results are consistent with those demonstrated in multicenter randomized phase IIIb MabEase study [12]. It was observed that scores for 'impact on activities of daily living", 'convenience', and 'satisfaction' in the questionnaire were improved with SC vs. IV administration of rituximab. Moreover, in this study QOL during SC rituximab treatment was better in four domains including psychological impact, convenience of therapy, impact on activities of daily living and satisfaction with therapy. There were no difference observed in physical impact [12]. The same results were observed in our study. QOL and physiological well-being was statistically better in SC administration in both FACT/GOG-Ntx and EORT-QLQ-C310.

An interesting findings of our study was that patients treated with rituximab SC had statistically less gastric problems than those treated with IV formula. So far, in majority of clinical studies comparing IV and SC administration, there were no difference in safety profile and frequency of side effects $[12,13]$. In our study we observed for the first time in the literature statistical difference in frequency of vomiting, nausea, and lack of appetite mentioned from our patients in questionnaire. It is very important that this findings were not published in any other study comparing SC and IV route od administration.
Also looking at economic side of SC administration, this type of dosing saves time and following that it has been calculated that it also decreases costs of overall treatment [14]. The use of rituximab SC resulted in substantial savings in clinic time. These findings concur with a time and motion analysis based on data collected within the MabCute study [15]. What is more, not only SC formula shorter dosing time but patients spend less time in a hospital so can preserve better state of being which is very important in long distance treatment. This was very important issue that was mentioned in questionnaire in majority of patients treated in our Outpatient Clinic. As it was observed in MabEase study shorter duration of administration caused that both nurses and doctors had "more than enough time" to discuss their treatment schedule and provide all answers to their questions [12]

The main limitation of our study is small group of patients. In future bigger group with similar B-NHL types should be planned to confirm the results of our study.

As it was already observed in case of safety profile and efficiency both types of administration represent comparable results. Moreover, it was noticed that SC formula of rituximab is much more preferred by patients with B-cell NHL. In conclusion, as it was observed in our study SC administration gives better satisfaction and QOL. These observation, together with data demonstrating the economic advantages the SC formula of rituximab should be always considered as the first way of treatment in DLBCL an FL patients. Moreover, it should be also possible option for all patients also with different diagnosis who are treated with rituximab. In near future it may be possible to administer SC formula at patients home, for those who will be qualified for this type of treatment in monotherapy.

\section{CONCLUSION}

The results from our study assessing $\mathrm{QOL}$ in lymphoma patients treated with SC vs. IV rituximab were similar to those from large multicenter clinical studies. The majority of patients felt more comfortable and were able to participate in social life. We found for the first time that SC administration is related with less gastric problems including vomiting, nausea and lack of appetite. 


\section{References}

1. Smith MR. Rituximab (monoclonal anti-CD20 antibody): mechanisms of action and resistance. Oncogene. 2003; 22: 7359-68.

2. Coiffier B. Long-term outcome of patients in the LNH-98.5 trial, the first randomized study comparing rituximab-CHOP to standard CHOP chemotherapy in DLBCL patients: a study by the Goupe d'Etudes des Lymphomes de l'Adulte. Blood. 2010; 116: 2040-5.

3. Davies A, Berge C, Boehnke A et al. Subcutaneous Rituximab for the treatment of B-cell hematologic malignancies: a review of the scientific rationale and clinical development. Adv Ther. 2017; 34: 2210-31.

4. Payandeh Z, Bahrami AA, Hoseinpoor R et al. The applications of anti-CD20 antibodies to treat various B cells disorders. Biomed Pharmacother. 2019; 109: 2415-26.

5. Bookbinder LH, Hofer A, Haller MF et al. A recombinant human enzyme for enhanced interstitial transport of therapeutics. J Control Release. 2006; 11 : 230-41.

6. Mao CP, Brovarney MR, Dabbaghet $\mathrm{K}$ et al. Subcutaneous versus intravenous administration of rituximab: pharmacokinetics, CD20 target coverage and B-cell depletion in cynomolgus monkeys. PLoS One. 2013; 8: e80533.

7. Davies A, Merli F, Mihaljevićet B et al. Efficacy and safety of subcutaneous rituximab versus intravenous rituximab for first-line treatment of follicular lymphoma (SABRINA): a randomised, open-label, phase 3 trial. Lancet Haematol. 2017; 4: e272-e82.

8. De Cock E, Kritikou P, Sandoval M et al. Time Savings with Rituximab Subcutaneous Injection versus Rituximab Intravenous Infusion: A Time and Motion Study in Eight Countries. PLoS One. 2016; 11: e0157957.

9. Delgado Sánchez O, Gutiérrez A, do Pazo F et al. Comparative Cost Analysis Of Intravenous And Subcutaneous Administration Of Rituximab In Lymphoma Patients. Clinicoecon Outcomes Res. 2019; 11: 695-701.

10. Pivot X, Verma S, Fallowfield L et al; PrefHer Study Group. Efficacy and safety of subcutaneous trastuzumab and intravenous trastuzumab as part of adjuvant therapy for HER2-positive early breast cancer: Final analysis of the randomised, two-cohort PrefHer study. Eur J Cancer. 2017; 86: 82-90.

11. Melichar B. PrefHer: finally addressing the preferences of her, too. Lancet Oncol. 2013; 14: 914-5.

12. Lugtenburg P, Avivi I, Berenschot $\mathrm{H}$ et al. Efficacy and safety of subcutaneous and intravenous rituximab plus cyclophosphamide, doxorubicin, vincristine, and prednisone in first-line diffuse large B-cell lymphoma: the randomized MabEase study. Haematologica. 2017; 102: 1913-22.

13. Rummel M, Kim TM, Aversa F et al. Preference for subcutaneous or intravenous administration of rituximab among patients with untreated CD20+ diffuse large B-cell lymphoma or follicular lymphoma: results from a prospective, randomized, open-label, crossover study (PrefMab). Ann Oncol. 2017; 28: 836-42

14. Sanchez Delgado O, Gutiérrez A, do Pazo F et al. Comparative Cost Analysis Of Intravenous And Subcutaneous Administration Of Rituximab In Lymphoma Patients. Clinicoecon Outcomes Res. 2019; 11:695-701.

15. Rule S, Barreto WG, Briones J et al. Efficacy and safety of prolonged maintenance with subcutaneous rituximab in patients with relapsed or refractory indolent NHL: results of the phase III MabCute study. Hematol Oncol. 2019; 37(S2): 229-31.

Authors' contributions:

Magdalena Witkowska: designed the research, analyzed the data, and wrote the paper; Sonia Witkowska: performed the research and analyzed and interpreted the data; Marika Klimczyk: performed the research and analyzed and interpreted the data; Anna Frydecka: performed the research and analyzed and interpreted the data; MichałWitkowski: collected the medical data and critically revised the manuscript; Piotr Smolewski: supervision.

Conflict of interests:

The authors declare no conflict of interest regarding the publication of this article.

Financial support:

The funders had no role in study design, data collection and analysis, decision to publish, or preparation of the manuscript.

Ethics:

The authors had full access to the data and take full responsibility for its integrity.

All authors have read and agreed with the content of the manuscript as written. The paper complies with the Helsinki Declaration, EU Directives and harmonized requirements for biomedical journals. 